\title{
Pancreatitis and pancreatic cancer in two large pooled case-control studies
}

\author{
Paige M. Bracci · Furong Wang · Manal M. Hassan • \\ Samir Gupta $\cdot$ Donghui Li $\cdot$ Elizabeth A. Holly
}

Received: 12 March 2009/Accepted: 25 August 2009/Published online: 17 September 2009

(C) The Author(s) 2009. This article is published with open access at Springerlink.com

\begin{abstract}
Objective The association between duration of pancreatitis and pancreatic cancer has not been well characterized in large population-based studies. We conducted detailed analyses to determine the association between pancreatitis onset and pancreatic cancer risk.

Methods Data from two case-control studies of pancreatic cancer $(n=4515)$ in the San Francisco Bay Area and the M.D. Anderson Cancer Center were pooled for analysis (1,663 cases, 2,852 frequency-matched controls). Adjusted odds ratios (OR) were estimated using a random-effects model.

Results In the pooled multivariable model, history of pancreatitis was associated with a 7.2-fold increased risk estimate for pancreatic cancer [95\% confidence interval (CI): 4.0, 13]. The risk estimate was nearly 10 -fold in
\end{abstract}

P. M. Bracci $(\bowtie) \cdot$ F. Wang · E. A. Holly

Department of Epidemiology and Biostatistics, School of

Medicine, University of California, 3333 California

St. Suite 280, San Francisco, CA 94143, USA

e-mail: paige.bracci@ucsf.edu

M. M. Hassan · D. Li

Department of Gastrointestinal Medical Oncology,

The University of Texas M.D. Anderson Cancer Center,

1515 Holcombe Blvd, Houston, TX 77030, USA

S. Gupta

Department of Internal Medicine, Division of Digestive and Liver Diseases, University of Texas Southwestern Medical Center, Dallas, TX, USA

E. A. Holly

Department of Health Research and Policy, Stanford University

School of Medicine, Stanford, CA 94305, USA participants aged $<55$ years ( $O R=9.9,95 \%$ CI: $3.5,28)$. A shorter temporal history of pancreatitis was more closely associated with pancreatic cancer than was a longer temporal history: $<3$ years $(\mathrm{OR}=29,95 \% \mathrm{CI}: 12,71), 3-10$ years $(\mathrm{OR}=2.6,95 \% \mathrm{CI}: 1.5,5.6)$, and $>10$ years $(\mathrm{OR}=1.8$, 95\% CI: $\left.0.7,4.5, p_{\text {trend }}<0.001\right)$.

Conclusion A short temporal history of pancreatitis was highly associated with pancreatic cancer, suggesting that pancreatitis may be an early manifestation of pancreatic cancer in some individuals. Pancreatic cancer should be considered in the differential diagnosis of individuals with an episode of pancreatitis.

Keywords Pancreatitis - Pancreatic Neoplasms . Inflammation · Case-control studies · Epidemiology

\section{Introduction}

Pancreatic cancer ranks fourth in cancer-related mortality in the United States with a 5-year survival rate of $<5 \%$ and incidence rates that are slightly higher in men than in women [1]. The etiology and biologic mechanisms of pancreatic cancer are not clear, but it is likely that medical conditions, genetic, and environmental factors are involved in tumor development as summarized in two recent reviews $[2,3]$ and in other investigations [4-9]. Cigarette smoking is one of the few established risk factors [10-12], and preliminary data suggest that this association may be modified for carriers of rare alleles in genes involved in the detoxification of polyaromatic hydrocarbons in tobacco smoke [5].

Pancreatitis is an inflammatory condition of the pancreas, traditionally classified as acute or chronic, although identification of an acute episode in patients 
with underlying chronic pancreatitis can be challenging and acute one-time episodes may go undiagnosed [13-16]. Alcohol is a dominant identified cause for chronic pancreatitis, particularly among men. Although a significant number of individuals may have chronic pancreatitis of idiopathic origin [14], recently published studies suggest that overall, alcohol may account for a smaller proportion of pancreatitis than in the earliest studies [17, 18]. Alcohol consumption has been attributed to pancreatitis in approximately $30-70 \%$ of men and in $0-15 \%$ of women, whereas women most commonly have idiopathic or biliary-tract associated pancreatitis [17, 19-26].

As pancreatic cancer may obstruct pancreatic enzyme flow, pancreatitis may be a consequence of pancreatic cancer. In studies with limited follow-up, up to $2 \%$ of individuals with acute pancreatitis have been reported to have pancreatic cancer [19, 20, 24-26]. However, upon exclusion of individuals diagnosed with pancreatitis in close proximity to pancreatic cancer, some have reported a continued strong association between a history of pancreatitis and/or chronic pancreatitis and pancreatic cancer, whereas others have not $[10,11,27-39]$. Given that inflammation has been implicated in the causal pathway of many other malignancies, possibly through increased cell proliferation due to chronic inflammation in the presence of growth factors [40-42], a causal relationship between pancreatitis and pancreatic cancer is plausible. Pancreatitis diagnosed in close temporal proximity to pancreatic cancer also may represent misdiagnosis of pancreatic cancer as pancreatitis. Research is needed to assess misdiagnosis and the magnitude of its effect upon risk factor analysis and to determine whether pancreatitis is an initiating step for pancreatic cancer or a consequence of pancreatic cancer.

Due to the aggressive nature of pancreatic cancer, a large percentage of patients in most population-based studies have died before they could be contacted. Very few studies have been large enough to analyze the temporal relationship between pancreatitis and pancreatic cancer, and results from these few retrospective cohort studies have been inconsistent likely due to differences in design and analysis methods [29, 30, 33, 35].

To better elucidate the association between pancreatitis and pancreatic cancer, data were pooled and analyzed from a population-based case-control study conducted in the San Francisco Bay Area and a hospital-based case-control study conducted at the M.D. Anderson Cancer Center. We hypothesized that pancreatitis in proximity to the diagnosis of pancreatic cancer would be associated with higher risk estimates for pancreatic cancer than would a diagnosis of pancreatitis that was made many years prior to the cancer diagnosis.

\section{Materials and methods}

Study populations

\section{The San Francisco Bay Area study}

Detailed methods have been published [5, 7, 9, 43-48]. Briefly, a population-based case-control study was conducted in the San Francisco Bay Area using rapid case ascertainment with a goal to identify patients in the study area within 1 month of diagnosis. Eligible patients were diagnosed between 1995 and 1999 with incident adenocarcinoma of the exocrine pancreas, were between 21 and 85 years old, resided in one of the six Bay Area counties, were alive at the time of first attempted contact, and could complete an interview in English. A total of 532 eligible cases completed the interview for a $67 \%$ response rate and $8 \%$ refusal rate $[5,7,9,43-48]$. Patient diagnoses were confirmed by the participants' physicians and by the Surveillance, Epidemiology, and End Results (SEER) abstracts that included histologic confirmation of disease. The eligibility criteria for controls were identical to those for cases except for pancreatic cancer status. Control participants were identified within the six San Francisco Bay Area counties using random-digit dial and were frequency-matched to cases by sex and by 5 -year agegroups for an approximate 1:3 case-to-control ratio. Control recruitment for those over $>65$ years of age was supplemented by random sampling of the Health Care Finance Administration (now the Centers for Medicare \& Medicaid Services) lists for the six Bay Area counties. A total of 1,701 eligible control participants completed the study interview for a response rate of $67 \%[5,7,9$, 43-48].

\section{The M.D. Anderson study}

A hospital-based case-control study of pancreatic cancer was conducted between January 2001 and May 2006 at the University of Texas M.D. Anderson Cancer Center (MDACC). The detailed methodology has been described previously $[49,50]$. Patients with pathologically confirmed adenocarcinoma of the pancreas were identified among patients who were evaluated and treated at the Gastrointestinal Cancer Clinic of the MDACC. Eligible cases were United States' residents, were able to communicate in English, and had no history of other cancers including other types of pancreatic cancer. Among 1,367 recruited patients, 236 were excluded because of missing epidemiological information, history of cancer other than non-melanoma skin cancer, or a pancreatic cancer diagnosis other than primary adenocarcinoma of the exocrine pancreas. A total of 1,131 cases with complete information were included in 
the final analyses. Controls were recruited from among individuals who accompanied cancer patients (nonpancreatic cancers) attending the institution's Central Diagnostic Radiology Clinics. Controls were not genetically related to the patients. They were the spouse, friends, and in-laws of patients with cancers other than gastrointestinal malignancies, lung cancer, and head and neck smoking-related cancers. Controls met the same criteria as cases except that they were cancer-free and were frequency matched to cases by age ( \pm 5 years), sex, and race/ethnicity. A total of 1,115 eligible controls ( $84 \%$ response rate) were included in the final analyses.

Study conduct and data collection of both studies

In both studies, trained interviewers conducted in-person interviews at the participants' homes or at a convenient location (for the San Francisco Bay Area Study) and at M.D. Anderson Cancer Center (for the MDACC Study). No proxy interviews were conducted at either site. Each study used a structured questionnaire that included demographic factors, smoking, alcohol, medical, occupational, and dietary history. Participants were asked if they ever had been diagnosed with pancreatitis by a physician, their age at diagnosis, the total number of episodes, whether they had a history of chronic pancreatitis, and their age when the last episode occurred. Because these last two pancreatitis characteristics were not asked in the MDACC study and numbers were too sparse for meaningful analyses in the UCSF San Francisco Bay Area dataset, they are not addressed in the analyses presented here.

Each study was reviewed and approved by its site-specific institutional review board, the University of California San Francisco (UCSF) and the University of Texas MDACC. In both studies, a written informed consent was obtained from participants prior to interview.

For both studies, age was categorized in 5-year agegroups for multivariable analyses. Data regarding potential confounding factors including cigarette smoking, alcohol consumption, diabetes mellitus, and gallbladder disease were collected in both studies. Smokers were defined as those participants who had smoked $>100$ cigarettes in their lifetime or had smoked a pipe or cigar at least once per month for $\geq 6$ months. Tobacco smoking was categorized into five groups based on lifetime history of smoking: never smokers; pipe and/or cigar smokers; former cigarette smokers who had quit $>15$ years before diagnosis (or interview for controls); former cigarette smokers who had quit 1-15 years before diagnosis/interview; and current smokers who by definition included those who quit within 1 year of diagnosis/interview. For stratified analyses, categories were dichotomized as never smokers versus ever smokers. Alcohol consumption for those who reported ever having consumed more than one drink per month of beer, wine, or liquor was computed as the average amount consumed weekly over the current and past two decades. One drink of alcohol was defined as one can, bottle, or $12 \mathrm{oz}$ glass of beer, or one $4-5 \mathrm{oz}$ glass of wine, or one shot of liquor given that each contains approximately the same amount of alcohol. Alcohol consumption was categorized as: 0 to $<1$ drink/week; $1-7$ drinks/week; 8-14 drinks/week; 15-21 drinks/week; and $>21$ drinks/week. The cut-point for the highest category was based on the top decile of alcohol consumption among controls. Analyses also were stratified by age $<55$ versus $\geq 55$ years to determine whether early pancreatic cancer onset [51] modified the association between pancreatitis and pancreatic cancer and to help identify a possible subgroup for early diagnosis.

To assess the temporal association between pancreatitis and pancreatic cancer, we computed duration as the difference in years between age first diagnosed with pancreatitis and age at pancreatic cancer diagnosis (or interview for controls). For analyses, duration was grouped as $<3$, $3-10$, and $>10$ years to distinguish pancreatitis that was more likely to have been associated with pancreatic cancer development from pancreatitis that was more likely due to other factors and for comparability with other published studies.

\section{Statistical methods}

Available data from each study were pooled using the STATA software program(version 9; Stata Corp, College Station, Texas). The crude associations between pancreatic cancer status and demographic characteristics and potential risk factors were assessed using Student's $t$-test for continuous variables and the chi-square test for categorical variables. Student's $t$-test also was used to test whether age at pancreatic cancer diagnosis was independent of history of pancreatitis. Analyses of the pooled data were conducted using multivariable unconditional logistic regression fixedeffects and random-effects models to compute odds ratios (OR) as estimates of the relative risk and $95 \%$ confidence intervals [52]. Between study heterogeneity was evaluated using the $Q$ and $I^{2}$ statistics [53]. Variables significant at $p<0.05$ in crude analyses were considered in the multivariable analyses. The final model that included age, sex, race, smoking (not in smoking stratified analyses in Table 2), alcohol consumption, and history of diabetes mellitus and gallbladder disease (not in gallbladder disease stratified analyses in Table 2) was selected based on biologic plausibility and the lowest -2 log likelihood statistic. Tests for trend for categorical variables were computed using a likelihood ratio test based on models that included the factor as an ordinal variable. Results were considered 
statistically significant for two-sided $p$-values less than 0.05 and somewhat significant for $p$-values $\geq 0.05$ and $\leq 0.10$.

\section{Results}

The frequency distributions of demographic characteristics and selected medical factors by case-control status were similar across the study populations (Table 1). In the pooled dataset, pancreatic cancer patients with a history of pancreatitis were younger at diagnosis than were study patients without pancreatitis (median age: 60 vs. 64 years, respectively, $p=0.001)$. Controls in both studies also were somewhat more likely to have higher levels of education compared with cases.
A total of 137 cases (9\%) and 28 controls (1\%) reported a history of pancreatitis (Table 2). Of these, $69 \%$ of cases and $71 \%$ of controls were former or current smokers (data not shown in table). History of pancreatitis was associated with a 7.2-fold increased risk estimate for pancreatic cancer in the pooled dataset (Table 2) and was consistent with the studyspecific estimates (UCSF: OR $=9.9,95 \%$ CI: 4.9-20; MDACC: $\mathrm{OR}=5.3$, 95\% CI: 2.9-9.5; data not in table). Risk estimates from multivariable stratified analyses (Table 2) were higher in men $(\mathrm{OR}=8.0)$ than in women $(\mathrm{OR}=6.8)$, in those with early-onset pancreatic cancer (age $<55$ years, $\mathrm{OR}=9.9$ ) compared with those with later-onset (age $\geq 55$ years, OR $=6.7$ ), in those without gallbladder disease $(\mathrm{OR}=9.7)$ compared with those with a history of gallbladder disease $(\mathrm{OR}=4.1, p$ for interaction $=0.02)$ and among non-smokers $(\mathrm{OR}=11)$ compared with smokers

Table 1 Demographic characteristics of study populations in case-control studies at the University of California San Francisco (UCSF) and the M.D. Anderson Cancer Center (MDACC), 1999-2006

\begin{tabular}{|c|c|c|c|c|c|c|c|c|}
\hline \multirow[t]{3}{*}{ Characteristics of study populations } & \multicolumn{4}{|c|}{ UCSF site } & \multicolumn{4}{|c|}{ MDACC site } \\
\hline & \multicolumn{2}{|c|}{ Cases $(n=532)$} & \multicolumn{2}{|c|}{ Controls $(n=1701)$} & \multicolumn{2}{|c|}{ Cases $(n=1131)$} & \multicolumn{2}{|c|}{ Controls $(n=1115)$} \\
\hline & No. ${ }^{\mathrm{a}}$ & $\%$ & No. ${ }^{\mathrm{a}}$ & $\%$ & No. $^{\mathrm{a}}$ & $\%$ & No. ${ }^{\mathrm{a}}$ & $\%$ \\
\hline \multicolumn{9}{|l|}{ Sex } \\
\hline Men & 291 & 55 & 883 & 52 & 651 & 58 & 682 & 61 \\
\hline Women & 241 & 45 & 818 & 48 & 480 & 42 & 433 & 39 \\
\hline \multicolumn{9}{|l|}{ Age (in years) } \\
\hline$<50$ & 46 & 9 & 164 & 10 & 138 & 12 & 190 & 17 \\
\hline $50-59$ & 120 & 22 & 438 & 25 & 311 & 27 & 324 & 29 \\
\hline $60-69$ & 172 & 33 & 473 & 28 & 402 & 36 & 375 & 34 \\
\hline $70-79$ & 158 & 29 & 498 & 30 & 254 & 23 & 207 & 19 \\
\hline$\geq 80$ & 36 & 7 & 128 & 8 & 26 & 2 & 19 & 2 \\
\hline \multicolumn{9}{|l|}{ Race/ethnicity } \\
\hline White & 423 & 80 & 1,367 & 80 & 972 & 86 & 995 & 89 \\
\hline Hispanic & 19 & 4 & 104 & 6 & 71 & 6 & 74 & 7 \\
\hline Black & 46 & 9 & 78 & 5 & 71 & 6 & 36 & 3 \\
\hline Asian/Pacific Islander & 35 & 7 & 119 & 7 & 16 & 1 & 7 & 1 \\
\hline Other & 9 & 2 & 33 & 2 & 1 & 0.1 & 3 & 0.3 \\
\hline \multicolumn{9}{|l|}{ Education } \\
\hline$<$ High school & 71 & 13 & 162 & 10 & 94 & 8 & 26 & 2 \\
\hline High school & 164 & 31 & 372 & 22 & 309 & 27 & 288 & 26 \\
\hline $1-4$ years of college & 200 & 38 & 754 & 44 & 510 & 45 & 602 & 54 \\
\hline Graduate school & 97 & 18 & 413 & 24 & 218 & 19 & 199 & 18 \\
\hline \multicolumn{9}{|l|}{ History of $>1$ year diabetes } \\
\hline Yes & 77 & 14 & 163 & 10 & 156 & 14 & 87 & 8 \\
\hline No & 455 & 86 & 1,538 & 90 & 975 & 86 & 1,028 & 92 \\
\hline \multicolumn{9}{|l|}{ Gallbladder disease } \\
\hline Yes & 99 & 19 & 207 & 12 & 255 & 23 & 127 & 11 \\
\hline No & 432 & 81 & 1,493 & 88 & 876 & 77 & 988 & 89 \\
\hline
\end{tabular}

${ }^{a}$ Numbers may not add up to the total number of participants because of missing values 
Table 2 Pooled adjusted odds ratios (OR) and $95 \%$ confidence intervals (CI) for history of pancreatitis and pancreatic cancer: University of California San Francisco (UCSF) and M.D. Anderson Cancer Center (MDACC) case-control studies, 1999-2006
History of pancreatitis ${ }^{\mathrm{a}}$

Pooled data UCSF \& MDACC

$P(Q$ test $)$

Cases/controls

$1,659 / 2,815$

Eve

No

Yes

Men

No

Yes

Women

No

Yes

OR $(95 \% \mathrm{CI})^{\mathrm{b}}$

Age at pancreatic cancer diagnosis or at interview for controls

$<55$ years

No

Yes

Yes

$>10$ years

$p$-Value for trend

No history of gallbladder diseases

$$
\text { No }
$$

Yes

History of gallbladder diseases

No

Yes

Never smokers

No

Yes

Ever smokers

No

Yes
$658 / 1238$

$62 / 13$

$1,522 / 2,787$

$137 / 28$

$864 / 1549$

$75 / 15$

$339 / 718$

$40 / 6$

$1183 / 2069$

$97 / 22$

$109 / 5$

$18 / 11$

$10 / 10$

$1218 / 2465$

$88 / 15$

$303 / 321$

$49 / 13$

$560 / 1185$

$43 / 8$

0.2

1.0

$7.2(4.0-13)$ $<0.001$

$962 / 1602$

$94 / 20$
1.0

$8.0(4.1-16)$

1.0

$6.8(3.1-15)$

1.0

$9.9(3.5-28)$

1.0

$6.7(2.3-20)$

29 (12-71)

0.5

$2.6(1.2-5.6)$

0.9

$1.8(0.7-4.5)$

0.9

1.0

$9.7(3.2-29)$

1.0

$4.1(2.0-108)$

0.8

1

$11(1.5-78)$

0.1

1

(1)

8

1

$6.8(4.1-11)$

smoking was 0.14

$(\mathrm{OR}=6.8, \quad p$ for interaction $=0.14)$. However, the estimates were based on a small number of exposed cases, and the associated confidence intervals were wide and overlapped for the stratified groups.

In analyses of the duration between pancreatitis and pancreatic cancer diagnoses (Table 2), 109 of 137 cases $(80 \%)$ and 5 of 28 controls $(18 \%)$ reported that their first episode of pancreatitis occurred $<3$ years before their pancreatic cancer diagnosis or interview (controls). ORs decreased with increasing duration, and the risk estimate for pancreatic cancer among those whose pancreatitis was diagnosed $<3$ years before their pancreatic cancer was substantially higher than among those whose pancreatitis onset occurred three or more years before their pancreatic cancer diagnosis. However, confidence intervals for the longer duration overlapped and included unity for duration of pancreatitis of $>10$ years (duration: $<3$ years, $\mathrm{OR}=29$; $3-10$ years, $\mathrm{OR}=2.6 ;>10$ years, $\mathrm{OR}=1.8$; trend $p<$ $0.0001)$. Sparse data prevented pooled analyses of duration stratified by sex and by early-onset (age $<55$ years).

\section{Discussion}

Our results demonstrate a marked strong association for pancreatic cancer with pancreatitis diagnosed within 
3 years prior to the pancreatic cancer diagnosis. In addition, modest increased risk estimates for pancreatic cancer that decreased over time were observed both for those with a diagnosis of pancreatitis 3-10 years and more than 10 years before their cancer diagnosis. The observed association may reflect pancreatitis as an initiator of pancreatic cancer and/or a consequence of pancreatic cancer. These results require confirmation and should be interpreted conservatively given that the CIs overlapped for the individual categories representing the longer latencies, and the $\mathrm{CI}$ for the longest duration category included unity. Our results also support further evaluation in larger pooled studies to determine whether other risk factors (sex, early onset, gallbladder disease, and smoking) modify the association between pancreatitis and pancreatic cancer and thus better define at-risk subgroups of patients.

Similar to our results, other studies that have examined the temporal association between pancreatitis and pancreatic cancer have reported an elevated risk estimate for pancreatic cancer diagnosed within the first few years following the diagnosis of pancreatitis [27, 29, 33, 35]. However, the differences in study designs and end-point measurements and the lack of mutually exclusive groups used in duration analyses have resulted in substantial variation in reported effects [27, 29, 33, 35]. Our results are consistent with those of a large Swedish retrospective cohort study of patients discharged with an initial diagnosis of pancreatitis between 1965 and 1983 [29, 33]. In this cohort study, the standardized incidence ratio (SIR) of pancreatic cancer decreased from 5.0 for 1-4 years of follow-up after pancreatitis diagnosis to 1.5 for 10-24 years of follow-up [33]. Interestingly, a persistent excess risk 10 years after pancreatitis was observed only among patients who also had been diagnosed as alcoholics [33]. Although our data also showed an increased risk estimate that persisted more than 10 years after pancreatitis diagnosis, the effect was not different from chance possibly due to the limited number of study participants in this category.

Our results differed from those reported for a medical records-based case-control study conducted through the department of Veteran Affairs (VA) in the US, although differences in computation of duration complicated the direct comparisons of results. Results from the VA study showed that ORs for pancreatic cancer decreased from 2.3 for pancreatitis diagnosed $\geq 1$ year before the diagnosis of pancreatic cancer to 2.0 for pancreatitis diagnosed 7-22 years before pancreatic cancer diagnosis [27]. However, because the VA analyses did not estimate risk using mutually exclusive categories for duration (e.g. mean length of follow-up was 8.2 years in the group followed up for $>1$ year), it is not possible from the published report to determine whether the observed decrease in their risk estimates is directly comparable to that observed in our study or in the Swedish study.

In contrast to our results, a hospital-based study in Italy reported that risk estimates for pancreatic cancer were higher for those whose duration between pancreatitis and pancreatic cancer diagnoses was five or more years compared with those whose duration was less than 5 years [30]. The duration cut-point of 5 years used in this Italian study prohibits a direct comparison of their results with ours and does not provide sufficient evidence to determine the effect of duration $>10$ years on pancreatic cancer risk estimates in their population. Similarly, the results from an international clinic/hospital-based study among patients with chronic pancreatitis estimated risk with person-years cumulated for $>2$ and $>5$ years since pancreatitis diagnosis [35]. Although there was some evidence among patients with non-alcoholic chronic pancreatitis that risk may decrease with increasing duration, long-term duration was not assessed [35].

Multiple possible explanations for the observed association between pancreatitis and pancreatic cancer have been suggested including misclassification of pancreatic cancer as pancreatitis, mechanical effects of the tumor due to infiltration of the pancreas and/or obstruction of the pancreatic duct, and inflammation. Differentiation of signs and symptoms of pancreatic disease due to pancreatic cancer and those due to pancreatitis can be challenging and may have affected the specificity of pancreatitis diagnosis more often in cases with pancreatic cancer than among controls, leading to differential misclassification of the pancreatitis history. Medical record confirmation was not obtained for patients who reported a physician-diagnosed episode of pancreatitis to support the specificity of the pancreatitis diagnosis. The effect of misclassification of pancreatitis diagnosis and the possible differential in misdiagnosis between cases and controls, if present, is unknown. If the observed associations were due to a mechanical effect related to underlying pancreatic cancer, pancreatitis would be a manifestation rather than a pathogenic step in the development of pancreatic cancer. Finally, inflammation may be due to the pancreatitis and thus a pathogenic step towards the development of pancreatic cancer, or it may be a consequence of the pancreatic cancer itself.

The inflammatory-related mechanisms that may cause, promote, or be a consequence of pancreatic cancer have not been established. Normal inflammation is limited by the release of anti-inflammatory cytokines. However, under conditions of chronic inflammation, the factors that control the inflammatory response are dysfunctional, thereby allowing inflammation to continue [41]. Chronic inflammation can promote DNA damage and cell proliferation via release of inflammatory mediators such as cytokines, chemokines, and reactive oxygen species. The inflammatory 
mediators that are commonly released during pancreatitis, NF-KB, IL-8, COX-2, and TNF-alpha, play a role in cell growth, proliferation, and apoptosis and also are highly expressed in pancreatic cancer [54]. The involvement of NF-KB is of particular interest as it is important in both inflammatory and oncogenic pathways [54]. Interestingly, in an earlier analysis of our data, we used DNA obtained from study participants to determine genetic susceptibility for pancreatic cancer associated with polymorphisms in inflammatory-related genes, including TNF-alpha and the chemokines, RANTES and CCR5 [55]. Our results showed that pancreatitis modified the association between pancreatic cancer and polymorphisms in TNF-alpha and RANTES such that risk estimates for pancreatic cancer were increased among those who carried the variant alleles for polymorphisms in these genes and also had a history of pancreatitis. The exact significance of these polymorphisms as markers of risk estimates and/or pathogenic factors requires validation and exploration in functional studies.

We investigated statistical interaction with other exposures and factors that have been associated with pancreatitis and with pancreatic cancer including gallbladder disease and smoking. Although intriguing, evidence to support that these factors modified the association between pancreatitis and pancreatic cancer was weak. Smoking and gallbladder/biliary disease may promote an inflammatory milieu that could contribute to pancreatitis and pancreatic cancer [56, 57]. Interestingly, gallbladder conditions have been associated with biliary pancreatitis which in turn has been correlated with acute rather than chronic pancreatitis [57]. This relationship may partly explain our observed higher risk of pancreatic cancer among pancreatitis patients without a history of gallbladder disease and with the lower risk among women, who are more likely than men to have biliary pancreatitis. However, our results suggesting that gallbladder disease history, smoking, and sex may modify the association between pancreatitis and risk of pancreatic cancer should be interpreted conservatively as they were based on a small number of exposed participants, were imprecise and remained elevated regardless of the factor level. The low frequency of pancreatitis limited our ability to conduct further detailed analyses but emphasizes the need to continue to investigate these complex associations in larger pooled studies.

One advantage of the current combined pooled analyses is the large sample size allowing for a detailed investigation of pancreatitis that was not possible in the individual studies. Additionally, participants in our two studies were interviewed in-person, thus minimizing the reporting bias associated with proxy interviews $[58,59]$. Further, we had a very low patient-refusal rate of $8 \%$ at the populationbased UCSF site and $6 \%$ at the clinic-based MDACC site, and the study hypotheses were unknown to the participants and to the interviewers.

Possible limitations common to case-control studies also should be considered when interpreting our results. Recall bias is possible but is likely to have been diminished by requesting that participants report "physician-diagnosed pancreatitis". Similar to other population-based studies of pancreatic cancer, a large number of patients had died prior to initial contact due to the aggressive nature of the disease, despite use of rapid case ascertainment methods at UCSF and enrollment of cases at diagnosis at MDACC. Comparison of SEER abstract demographic data for interviewed and non-interviewed cases identified as part of the UCSF study showed that non-interviewed cases tended to be slightly older, with a slightly greater proportion of women than men, somewhat more minorities, fewer known tumor characteristics, and a shorter survival time [46]. It is possible that cases diagnosed in proximity to their pancreatic cancer diagnosis may have incorrectly reported their pancreatitis diagnosis. Although this misclassification of pancreatitis may have been differential between cases and controls and resulted in an overestimate of the association with pancreatic cancer in the years closest to their cancer diagnosis, the practical implications of our results remain clinically important. Given the magnitude of the risk estimate for the shortest duration and the consistently elevated risks for longer duration, the association of long-term pancreatitits with pancreatic cancer risk requires further exploration.

In conclusion, results from our analyses in this large pooled dataset provide support for the hypothesis that some pancreatitis may be a clinical marker for early manifestation of pancreatic cancer or a pathogenic step towards the development of pancreatic cancer. Further investigation in larger pooled studies is required to confirm our results and to assess the joint effects with other risk factors that may contribute to pancreatic inflammation such as smoking and gallbladder disease. In addition, larger pooled analyses that include multiple case-control studies are needed to determine whether additional characteristics of pancreatitis such as age at pancreatitis onset, the number of episodes, chronic or acute disease, duration by sex, and family history of pancreatitis are related to the observed association with pancreatic cancer.

Acknowledgments Grant Support: This work was supported in part by National Cancer Institute grants CA59706, CA108370, CA109767, CA89726 (E.A. Holly, PI), and CA98380 (Li D.: PI), by the National Institutes of Health grant number 1 KL2 RR024983-01 from the National Center for Research Resources (NCRR), and by the Rombauer Pancreatic Cancer Research Fund, and The Lockton Pancreatic Cancer Research Fund. The collection of cancer incidence data for the UCSF study was supported by the California Department of Public Health as part of the statewide cancer reporting program mandated by California Health and Safety Code Sect. 103885; the National Cancer 
Institute's Surveillance, Epidemiology and End Results Program under contract N01-PC-35136 awarded to the Northern California Cancer Center; and the Centers for Disease Control and Prevention's National Program of Cancer Registries, under agreement \#U55/ CCR921930-02 awarded to the Public Health Institute. The ideas and opinions expressed herein are those of the author(s) and endorsement by the State of California, Department of Public Health the National Cancer Institute, and the Centers for Disease Control and Prevention or their Contractors and Subcontractors is not intended nor should be inferred.

Open Access This article is distributed under the terms of the Creative Commons Attribution Noncommercial License which permits any noncommercial use, distribution, and reproduction in any medium, provided the original author(s) and source are credited.

\section{References}

1. Jemal A, Siegel R, Ward E, Hao Y, Xu J, Thun MJ (2009) Cancer statistics, 2009. CA Cancer J Clin 59(4):225-249 Epub 2009 May 27

2. Anderson KE, Mack TM, Silverman DT (2006) Cancer of the pancreas. In: Schottenfeld D, Fraumeni JF (eds) Cancer epidemiology and prevention, 3rd edn. Oxford University Press, New York, pp 721-763

3. Duell EJ, Bracci PM, Holly EA (2005) Environmental determinants of exocrine pancreatic cancer. In: Pour PM (ed) Toxicology of the pancreas, 1st edn. Taylor \& Francis, New York, pp 395-422

4. Boyle P, Hsieh CC, Maisonneuve P et al (1989) Epidemiology of pancreas cancer (1988). Int J Pancreatol 5(4):327-346

5. Duell EJ, Holly EA, Bracci PM, Liu M, Wiencke JK, Kelsey KT (2002) A population-based, case-control study of polymorphisms in carcinogen-metabolizing genes, smoking, and pancreatic adenocarcinoma risk. J Natl Cancer Inst 94(4):297-306

6. Flanders TY, Foulkes WD (1996) Pancreatic adenocarcinoma: epidemiology and genetics. J Med Genet 33(11):889-898

7. Hoppin JA, Tolbert PE, Holly EA et al (2000) Pancreatic cancer and serum organochlorine levels. Cancer Epidemiol Biomarkers Prev 9(2):199-205

8. Lowenfels AB, Maisonneuve P (2002) Epidemiologic and etiologic factors of pancreatic cancer. Hematol Oncol Clin North Am $16(1): 1-16$

9. Slebos RJ, Hoppin JA, Tolbert PE et al (2000) K-ras and p53 in pancreatic cancer: association with medical history, histopathology, and environmental exposures in a population-based study. Cancer Epidemiol Biomarkers Prev 9(11):1223-1232

10. Farrow DC, Davis S (1990) Risk of pancreatic cancer in relation to medical history and the use of tobacco, alcohol and coffee. Int J Cancer 45(5):816-820

11. Kalapothaki V, Tzonou A, Hsieh CC, Toupadaki N, Karakatsani A, Trichopoulos D (1993) Tobacco, ethanol, coffee, pancreatitis, diabetes mellitus, and cholelithiasis as risk factors for pancreatic carcinoma. Cancer Causes Control 4(4):375-382

12. Silverman DT, Dunn JA, Hoover RN et al (1994) Cigarette smoking and pancreas cancer: a case-control study based on direct interviews. J Natl Cancer Inst 86(20):1510-1516

13. Sarner M, Cotton PB (1984) Definitions of acute and chronic pancreatitis. Clin Gastroenterol 13(3):865-870

14. Steer ML, Waxman I, Freedman S (1995) Chronic pancreatitis. N Engl J Med 332(22):1482-1490

15. Steinberg W, Tenner S (1994) Acute pancreatitis. N Engl J Med 330(17):1198-1210
16. Talamini G, Falconi M, Bassi C, Mastromauro M, Salvia R, Pederzoli P (2000) Chronic pancreatitis: relationship to acute pancreatitis and pancreatic cancer. Jop 1(3 Suppl):69-76

17. Frulloni L, Gabbrielli A, Pezzilli R et al (2009) Chronic pancreatitis: report from a multicenter Italian survey (PanCroInfAISP) on 893 patients. Dig Liver Dis. 41(4):311-317 Epub 2008 Dec 20

18. Whitcomb DC, Yadav D, Adam S et al (2008) Multicenter approach to recurrent acute and chronic pancreatitis in the United States: the North American pancreatitis study 2 (NAPS2). Pancreatology 8(4-5):520-531 Epub 2008 Sep 3

19. Appelros S, Borgstrom A (1999) Incidence, aetiology and mortality rate of acute pancreatitis over 10 years in a defined urban population in Sweden. Br J Surg 86(4):465-470

20. Gullo L, Migliori M, Olah A et al (2002) Acute pancreatitis in five European countries: etiology and mortality. Pancreas 24(3):223-227

21. Jaakkola M, Nordback I (1993) Pancreatitis in Finland between 1970 and 1989. Gut 34(9):1255-1260

22. Lankisch PG, Assmus C, Lehnick D, Maisonneuve P, Lowenfels AB (2001) Acute pancreatitis: does gender matter? Dig Dis Sci 46(11):2470-2474

23. Lin Y, Tamakoshi A, Matsuno S et al (2000) Nationwide epidemiological survey of chronic pancreatitis in Japan. J Gastroenterol 35(2):136-141

24. Maes B, Hastier P, Buckley MJ et al (1999) Extensive aetiological investigations in acute pancreatitis: results of a 1-year prospective study. Eur J Gastroenterol Hepatol 11(8):891-896

25. Pezzilli R, Billi P, Morselli-Labate AM (1998) Severity of acute pancreatitis: relationship with etiology, sex and age. Hepatogastroenterology 45(23):1859-1864

26. Thomson SR, Hendry WS, McFarlane GA, Davidson AI (1987) Epidemiology and outcome of acute pancreatitis. Br J Surg 74(5):398-401

27. Bansal P, Sonnenberg A (1995) Pancreatitis is a risk factor for pancreatic cancer. Gastroenterology 109(1):247-251

28. Bueno de Mesquita HB, Maisonneuve P, Moerman CJ, Walker AM (1992) Aspects of medical history and exocrine carcinoma of the pancreas: a population-based case-control study in The Netherlands. Int J Cancer 52(1):17-23

29. Ekbom A, McLaughlin JK, Karlsson BM et al (1994) Pancreatitis and pancreatic cancer: a population-based study. J Natl Cancer Inst 86(8):625-627

30. Fernandez E, La Vecchia C, Porta M, Negri E, d'Avanzo B, Boyle P (1995) Pancreatitis and the risk of pancreatic cancer. Pancreas 11(2):185-189

31. Gold EB, Gordis L, Diener MD et al (1985) Diet and other risk factors for cancer of the pancreas. Cancer 55(2):460-467

32. Jain M, Howe GR, St Louis P, Miller AB (1991) Coffee and alcohol as determinants of risk of pancreas cancer: a case-control study from Toronto. Int J Cancer 47(3):384-389

33. Karlson BM, Ekbom A, Josefsson S, McLaughlin JK, Fraumeni JF Jr, Nyren O (1997) The risk of pancreatic cancer following pancreatitis: an association due to confounding? Gastroenterology 113(2):587-592

34. La Vecchia C, Negri E, D'Avanzo B et al (1990) Medical history, diet and pancreatic cancer. Oncology 47(6):463-466

35. Lowenfels AB, Maisonneuve P, Cavallini G et al (1993) Pancreatitis and the risk of pancreatic cancer. International pancreatitis study group. N Engl J Med 328(20):1433-1437

36. Mack TM, Yu MC, Hanisch R, Henderson BE (1986) Pancreas cancer and smoking, beverage consumption, and past medical history. J Natl Cancer Inst 76(1):49-60

37. Malka D, Hammel P, Maire F et al (2002) Risk of pancreatic adenocarcinoma in chronic pancreatitis. Gut 51(6):849-852 
38. Partanen TJ, Vainio HU, Ojajarvi IA, Kauppinen TP (1997) Pancreas cancer, tobacco smoking and consumption of alcoholic beverages: a case-control study. Cancer Lett 116(1):27-32

39. Talamini G, Falconi M, Bassi C et al (1999) Incidence of cancer in the course of chronic pancreatitis. Am J Gastroenterol 94(5):1253-1260

40. Balkwill F, Mantovani A (2001) Inflammation and cancer: back to Virchow? Lancet 357(9255):539-545

41. Coussens LM, Werb Z (2002) Inflammation and cancer. Nature 420(6917):860-867

42. Farrow B, Evers BM (2002) Inflammation and the development of pancreatic cancer. Surg Oncol 10(4):153-169

43. Chan JM, Wang F, Holly EA (2005) Vegetable and fruit intake and pancreatic cancer in a population-based case-control study in the San Francisco Bay area. Cancer Epidemiol Biomarkers Prev 14(9):2093-2097

44. Duell EJ, Holly EA (2005) Reproductive and menstrual risk factors for pancreatic cancer: a population-based study of San Francisco Bay area women. Am J Epidemiol 161(8):741-747

45. Duell EJ, Holly EA, Bracci PM, Wiencke JK, Kelsey KT (2002) A population-based study of the Arg399Gln polymorphism in $\mathrm{X}$-ray repair cross-complementing group 1 (XRCC1) and risk of pancreatic adenocarcinoma. Cancer Res 62(16):4630-4636

46. Eberle CA, Bracci PM, Holly EA (2005) Anthropometric factors and pancreatic cancer in a population-based case-control study in the San Francisco Bay area. Cancer Causes Control 16(10):12351244

47. Holly EA, Eberle CA, Bracci PM (2003) Prior history of allergies and pancreatic cancer in the San Francisco Bay area. Am J Epidemiol 158(5):432-441

48. Wang F, Gupta S, Holly EA (2006) Diabetes mellitus and pancreatic cancer in a population-based case-control study in the San Francisco Bay area, California. Cancer Epidemiol Biomarkers Prev 15(8):1458-1463
49. Hassan MM, Abbruzzese JL, Bondy ML et al (2007) Passive smoking and the use of noncigarette tobacco products in association with risk for pancreatic cancer: a case-control study. Cancer 109(12):2547-2556

50. Hassan MM, Bondy ML, Wolff RA et al (2007) Risk factors for pancreatic cancer: case-control study. Am J Gastroenterol 102(12):2696-2707 Epub 2007 Aug 31

51. Couch FJ, Johnson MR, Rabe KG et al (2007) The prevalence of BRCA2 mutations in familial pancreatic cancer. Cancer Epidemiol Biomarkers Prev 16(2):342-346

52. DerSimonian R, Laird N (1986) Meta-analysis in clinical trials. Control Clin Trials 7(3):177-188

53. Higgins JP, Thompson SG (2002) Quantifying heterogeneity in a meta-analysis. Stat Med 21(11):1539-1558

54. Farrow B, Sugiyama Y, Chen A, Uffort E, Nealon W, Mark Evers B (2004) Inflammatory mechanisms contributing to pancreatic cancer development. Ann Surg 239(6):763-769 Discussion 769-71

55. Duell EJ, Casella DP, Burk RD, Kelsey KT, Holly EA (2006) Inflammation, genetic polymorphisms in proinflammatory genes TNF-A, RANTES, and CCR5, and risk of pancreatic adenocarcinoma. Cancer Epidemiol Biomarkers Prev 15(4):726-731

56. Greer JB, Whitcomb DC (2009) Inflammation and pancreatic cancer: an evidence-based review. Curr Opin Pharmacol 7:7

57. Lankisch PG, Breuer N, Bruns A, Weber-Dany B, Lowenfels AB, Maisonneuve P (2009) Natural history of acute pancreatitis: a long-term population-based study. Am J Gastroenterol 14:14

58. Nelson LM, Longstreth WT Jr, Koepsell TD, Checkoway H, van Belle G (1994) Completeness and accuracy of interview data from proxy respondents: demographic, medical, and life-style factors. Epidemiology 5(2):204-217

59. Nelson LM, Longstreth WT Jr, Koepsell TD, van Belle G (1990) Proxy respondents in epidemiologic research. Epidemiol Rev $12: 71-86$ 